\title{
Asma bronquial y ejercicio físico. Manifestaciones en el
}

\section{adulto mayor}

\section{Bronchial asthma and physical exercise. Manifestations in the elderly}

Adriana Mederos González. ${ }^{1}$ \& Lic. Eddy Herrera Fraga. ${ }^{2}$

\section{Recibido: 07-01-2019 / Revisado: 09-02-2019 / Aceptado: 11-03-2019 / Publicado: 04-04-2019}

\section{DOI: https://doi.org/10.33262/anatomiadigital.v2i2.1079}

\begin{abstract}
.
The global prevalence of asthma varies between $1 \%$ and $18 \%$ depending on the country, even within the same country it varies from region to region and depending on the ethnic group analyzed. The prevalence traditionally accepted in our country is $8.2 \%$, slightly higher in women than in men and in individuals from urban and coastal areas.

It is more frequent among children than among adults, with a male / female ratio of approximately 1.5 in childhood, $\mathrm{q}$ in late adolescence and below, and 1 during adulthood. The maximum values, even with interregional differences, come to be detected in the rich populations and those of AngloSaxon origin in both hemispheres, as well as certain countries in Central and South America.
\end{abstract}

\section{Resumen.}

La prevalencia global de asma varía entre $1 \%$ y $18 \%$ según países, incluso dentro de un mismo país varía de unas regiones a otras y en función del grupo étnico analizado. La prevalencia tradicionalmente aceptada en nuestro país es de $8.2 \%$, ligeramente superior en el sexo femenino que en el masculino y en individuos de zonas urbanas y costeras.

Resulta más frecuente entre los niños que entre los adultos, con una relación varón/mujer aproximada de 1.5 en la infancia, q en la adolescencia tardía e inferior y 1 durante la edad adulta. Los valores máximos, incluso con diferencias interregionales, vienen a detectarse en las poblaciones ricas y de origen anglosajón de ambos hemisferios, así como determinados países de Centroamérica y Suramérica.

\footnotetext{
${ }^{1}$ Ciencias Médicas Mayabeque, Mayabeque, Cuba, amederos@infomed.sld.cu 0002-7353-0143

${ }^{2}$ Ciencias Médicas Mayabeque, Mayabeque, Cuba, eherrera@infomed.sld.cu iD https://orcid.org/0000-00027353-0144

https://orcid.org/0000-
} 
Keywords: global prevalence, bronchial asthma, exercise.
Palabras claves: prevalencia global, asma bronquial, ejercicio.

\section{Introducción.}

El asma es una enfermedad inflamatoria crónica de la vía aérea, en cuya patogenia intervienen diferentes células y mediadores inflamatorios, condicionada parcialmente por factores genéticos, que cursa con hiperreactividad de la vía aérea a una gran variedad de estímulos y con obstrucción reversible del flujo aéreo, bien espontáneamente, bien con tratamiento broncodilatador. Esta inflamación causa episodios recurrentes de sibilancias, disnea, opresión torácica y tos. Es un síndrome con fenotipos diferentes que comparten unas manifestaciones clínicas similares, pero con etiologías probablemente distintas.

\section{Historia}

Desde los momentos más remotos de la historia de la medicina, los grandes maestros como Hipócrates, Avicena y otros señalaron la importancia del aire para la vida y señalaron los rudimentos de la fisiología pulmonar.

El asma bronquial ha ocupado ininterrumpidamente la atención médica desde la antigüedad (460-130 a.n.e), fue referida por Hipócrates, Galeno y Areteo de Capadocia. Celso en el año 30 a.n.e le dio como nombre "falta de aire moderada" que presentaban los soldados al hacer ejercicios.

Desde esos momentos hasta hoy en día, esta condición respiratoria despierta el mayor interés en todo el mundo, a pesar de la ausencia de una definición precisa de la enfermedad, es uno de los problemas mayores en estudio y atención a los pacientes que la sufren. El primer agente causal sigue siendo desconocido por lo que se eleva su dificultad, la anatomía patológica es de difícil obtención y las manifestaciones clínicas son variables y no pocas veces atípicas, lo que precisamente sucede en el asma; por tanto, esta es una enfermedad fácil de reconocer, pero difícil de definir.

\section{Epidemiologia}

Afecta a más de 300 millones de personas en todo el mundo.

- Prevalencia: la prevalencia global de asma varía entre $1 \%$ y $18 \%$ según países, incluso dentro de un mismo país varía de unas regiones a otras y en función del grupo étnico analizado. La prevalencia tradicionalmente aceptada en nuestro país es de $8.2 \%$, ligeramente superior en el sexo femenino que en el masculino y en individuos de zonas urbanas y costeras. 
Resulta más frecuente entre los niños que entre los adultos, con una relación varón/mujer aproximada de 1.5 en la infancia, q en la adolescencia tardía e inferior y 1 durante la edad adulta. Los valores máximos, incluso con diferencias interregionales, vienen a detectarse en las poblaciones ricas y de origen anglosajón de ambos hemisferios, así como determinados países de Centroamérica y Suramérica.

- Mortalidad: en todo el mundo se producen 250000 muertes por asma al año, no existe relación entre la prevalencia y la mortalidad por asma.

\section{Causas}

De entre las posibles causas consideradas, la que hoy goza de mayor predicamento es la vinculada con la exposición a ciertos agentes infecciosos (hipótesis de la higiene).

En síntesis, la hipótesis establece que determinadas infecciones comunes adquiridas en las primeras etapas del desarrollo pueden prevenir la aparición de la atopia; el origen del problema radica en la reducción de esas infecciones entre los miembros más jóvenes de las familias, dicha reducción es consecuencia de la mejoría de los estándares de vida, los programas de inmunización y/o el uso indiscriminado de antibióticos. Hay pruebas crecientes de que el contexto inmunológico durante la fase uterina se encuentra decantado de forma natural hacia el fenotipo linfocitos $\mathrm{T}$ belper 2 (Th2) posiblemente para proteger la placenta frente a los efectos tóxicos de las citosinas Th1, se mantiene así durante un cierto tiempo tras el nacimiento, generando una "ventana de alto riesgo" para la sensibilización alérgica temprana. El cierre de la "ventana" solo tendrá lugar si el individuo entra en contacto con microorganismos que, como los virus o los comensales del aparato digestivo, estimulan la respuesta inmune del tipo Th1 y equilibran la relación Th1/Th2. La ausencia de exposición ocasionará en definitiva que las respuestas de tipo Th2 frente a antígenos no microbianos queden facilitadas en los sujetos predispuestos genéticamente.

\section{Historia natural}

Por lo general, el asma comienza en las primeras etapas de la vida. En casi el 50\% de los casos, el pico de inicio se localiza por debajo de los 10 años de edad, y en mayor parte de los niños los síntomas aparecen antes de cumplir los 2 años.

Durante la adolescencia, la incidencia de asma decrece para volver a repuntar de nuevo al principio de la edad adulta. Sólo en menos del 25\% de los individuos, el diagnóstico se establece después de los 40 años. No obstante, en algunos de ellos es posible identificar una sintomatología respiratoria previa, e incluso anormalidades espirográficas; muchos de esos sujetos han sido etiquetados de "broncoquísticos crónicos" antes de ser categorizados de forma definitiva como asmáticos. 
Adopta un carácter agudo e intermitente en el 70-75\% de los niños que la padecen; la duración y gravedad de la crisis son variables y la función respiratoria tiende a volver a la normalidad entre los accesos. Por el contrario, en algo menos del 25\% de los casos de asma infantil, los episodios de sibilancias acostumbran a ser más prolongadas y frecuentes, y en un pequeño número de pacientes el proceso adquiere características de cronicidad y gravedad.

Con el comienzo de la edad adulta, una gran proposición de asmáticos, aunque la recidiva posterior puede ocurrir. Las probabilidades de que la enfermedad persista aumentan en función de la gravedad del asma en la infancia o si esta se acompañó de sinusitis crónica, pólipos nasales o eccema.

De entre los niños con asma leve e intermitente, más del $60 \%$ se encontrarían asintomáticos a los 21 años; en el resto; las sibilancias persistirán de forma ocasional. Sin embargo, en el grupo con sibilancias frecuentes, el $40 \%$ estará sustancialmente mejor a esa edad. Alrededor del $45 \%$ mantendrán su situación clínica y el 15\% empeorará. Cuando el asma comienza en la edad adulta tiene un peor pronóstico, y son menores de las variaciones espontáneas y la posibilidad de remisiones prolongadas. Si suceden, las recaídas resultan mucho más frecuentes que en el asma infantil, máxime cuando el paciente mantiene síntomas respiratorios residuales.

\section{Patogenia}

Es un trastorno inflamatorio crónico de las vías áreas. La inflamación crónica se asocia a hiperreactividad bronquial y a obstrucción reversible, aunque los mecanismos que explican esta asociación no están completamente aclarados. El proceso inflamatorio es bastante consistente entre pacientes y entre diferentes momentos evolutivos de la enfermedad.

La inflamación en el asma es similar a otros procesos alérgicos. Los factores implicados en la diátesis inflamatoria se pueden clasificar en los tres aparatos siguientes:

\section{Células inflamatorias:}

Linfocitos T: elevados en la vía aérea, con un aumento de las células natural killer.

Mastocitos: aumentados tanto en la vía aérea como en como en el músculo liso bronquial, lo que reacciona con la hiperreactividad bronquial. Su activación da lugar a la liberación de mediadores broncodilatadores y proinflamatorios.

Eosinófilos: elevados en la vía aérea de la mayoría de los asmáticos y su número se relaciona con la gravedad del asma. Su activación libera enzimas responsables del daño epitelial y mediadores que amplifican la respuesta inflamatoria. 
Neutrófilos: aparecen en cantidad aumentada en la vía aérea de algunos pacientes con asma grave, en exacerbaciones, en asmáticos fumadores y en algunos casos de asma profesional.

Células dendríticas: estimulan el desarrollo de linfocitos Th2.

Macrófagos: contienen receptores de baja afinidad para la $\operatorname{IgE}$ que cuando son estimulados por alérgenos liberan mediadores que amplifican la respuesta inflamatoria.

Células y elementos estructurales de la vía aérea:

Epitelio bronquial: el daño en el epitelio de la vía aérea es una característica del asma no controlada, con pérdida de células ciliadas y secretoras. A la vez interviene activamente en el proceso inflamatorio, liberando mediadores proinflamatorios en relación con diferentes estímulos que contribuyen a aumentar más el daño epitelial. El proceso de reparación de estas lesiones es anómalo, dando lugar a un componente de obstrucción irreversible que en ocasiones aparece en el asma, en un proceso conocido como remodelado de la vía aérea.

Músculo liso bronquial: contribuye a la obstrucción al flujo aéreo por su hipertrofia, contracción aumentada e incluso liberación de mediadores inflamatorios igual que las células epiteliales.

Células endoteliales: la expresión de moléculas de adhesión en las células del endotelio de la circulación bronquial es necesaria para el reclutamiento de células inflamatorias en la vía aérea.

Fibroblastos y miofibroblastos: estimulados por mediadores inflamatorios y factores de crecimiento, contribuyen mediante la producción anómala de tejido fibroso al remodelado de la vía aérea.

Terminaciones nerviosas colinérgicas de las vías aéreas: si se activan por vía refleja, causan broncoconstricción y secreción de moco. Producen síntomas como la tos y la opresión pericordal.

\section{Moléculas:}

Quimiotoxinas: expresadas por las células epiteliales, actúan reclutando células inflamatorias en las vías aéreas.

Cistenil-leucotrienos: sustancias liberadas por mastocitos y eosinófilos con fuerte efecto broncoconstrictor.

Citosinas: producidas por linfocitos y macrófagos, controlan el proceso inflamatorio en el asma y probablemente determinan su gravedad, las más importantes son las derivadas de los 
linfocitos Th2; interleucina IL-5, favorece la producción y activación de eosinófilos, y la IL4 e IL-3, necesarias para la producción de IgE por los linfocitos B.

IgE: inmunoglobulina responsable de los fenómenos alérgicos, se une a receptores de alta afinidad presentes en mastocitos, eosinófilos y células dendríticas. En presencia del alérgeno específico esta unión desencadena la liberación de mediadores en dichas células.

Óxido nítrico: potente vasodilatador producido en el epitelio bronquial por la acción de la enzima óxido nítrico-sintetasa. Su determinación en el aire espirado se puede emplear como prueba diagnóstica, por ser un marcador no invasivo de la vía aérea.

\section{Fisiopatología}

Las alteraciones estructurales de la mucosa y de la pared bronquial dan como resultado dos consecuencias: la obstrucción y la hiperrespuesta de la vía aérea ante estímulos contracturantes (metacolina, adenosina, etc.). La obstrucción, difusa pero no uniforme, condiciona una serie de modificaciones en la mecánica respiratoria, con cambios sustanciales en las relaciones entre la profusión, flujo y volumen pulmonar que pueden llegar a traducirse en una alteración de la relación ventilación/perfusión y del intercambio gaseoso.

La hiperrespuesta se origina por una suma de factores. El aumento de espesor de las diferentes capas de la vía aérea o la reducción de las cargas elásticas debidas a los cambios en la matriz del tejido conectivo provocan que, ante un mismo grado de acortamiento del MLVA, la reducción de la luz bronquial sea mayor. Además, el MLVA del asmático tiene una mayor sensibilidad frente a los agentes bronco constrictores, ya que los mediadores inflamatorios determinan un incremento de la disponibilidad del mensajero intracelular regulador de su tono: e Ca2+ intracitoplasmático. Para otros, la hiperrespuesta es el resultado, sobre todo, de la pérdida de algún factor operativo en los individuos normales encargado de modular y limitar la contractilidad del miocito. El dilatador endógeno sería la propia respiración y el estiramiento clínico a que se ve sometido el músculo de la pared bronquial con los cambios de volumen pulmonar. El fracaso de ese mecanismo broncoprotector, tal vez relacionado con un aumento en la longitud de los filamentos de actina y un comportamiento del MLVA más elástico que plástico, condicionaría finalmente una mayor capacidad de contracción y una mayor velocidad de acortamiento.

\section{Manifestaciones clínicas}

El diagnostico de asma se instaura combinando la historia clínica y la funcional respiratoria, los síntomas típicos son tos, disnea, sibilancias audibles y opresión torácica, de carácter variable y en diferentes combinaciones, con predominio nocturno o durante las primeras horas de la mañana. El 80\% de los asmáticos alérgicos y el 50\% de los no alérgicos refieren también clínica rinosinusal. La anamnesis rastreará igualmente la presencia o ausencia de 
antecedentes familiares y personales de atopía y/o asma. La espirometría y las pruebas broncomotoras permitirán finalmente confirmar o descartar el diagnóstico de sospecha. La fluctuación de la función pulmonar en el tiempo, incluso en un mismo día, es una característica de esa entidad que puede observarse con la medida diaria del flujo respiratorio máximo. Por otro lado, la utilidad práctica de marcadores de inflamación (óxido nítrico en el aire exhalado o determinación de eosinófilos en el esputo) está aún por establecer con exactitud, aunque, con razonable seguridad, una clínica compatible y valores de óxido nítrico en el aire espirado superiores a 30 partes por billón en no fumadores son muy sugestivos de la enfermedad asmática. Una vez establecido el diagnóstico, el estudio del perfil alérgico, sospechado a partir de la historia clínica, se establecerá mediante las pruebas cutáneas de punción epidérmica en prick o midiendo la IgE específica circulante. La selección de los aeroalergenos sospechosos (polen, ácaros, epitelio de animales, etc.) variaría según la zona específica.

\section{Medidas preventivas}

La eliminación total o parcial de los alérgenos más comunes del entorno del asmático plantea diversas dificultades, aunque debe siempre intentarse. Asimismo, es importante evitar el tabaquismo activo o pasivo y la exposición a los alérgenos y sustancias irritantes existentes en el lugar de trabajo que empeoran el asma, de no ser posible, con el cambio de actividad laboral. Algunos asmáticos, en especial los que cursan con poliposis nasosinusal, desarrollan broncoespasmo grave cuando toman ácido acetilsalicílico u otros antiinflamatorios no esteroideos (AINE). Es pues capital que otros enfermos sean correctamente diagnosticados, ya sea mediante una historia clínica (antecedentes de reacciones a distintos AINE) o mediante provocación oral o nasal. Los intolerantes a AINE que requieran analgesia deben tratarse con paracetamol sin que la dosis exceda los $650 \mathrm{mg}$. Para una analgesia más potente, cabe recurrir, de forma progresiva, a asociación de codeína y paracetamol, el uso de dextropropoxifeno o la administración de pentazocina y derivados mórficos. Cuando se necesite de un tratamiento antiinflamatorio, pueden emplearse como alternativa los inhibidores selectivos (meloxicam) o específicos de la ciclooxigenasa-2 (etoricoxib y celecoxib) confirmado previamente la tolerancia a los mismos.

\section{Tratamiento farmacológico}

Los antiasmáticos disponibles se agrupan bajo dos grandes categorías: los fármacos de mantenimiento y los fármacos de rescate. Los primeros (glucocorticoides inhalados y sistémicos, antagonistas de los LT, agonistas adrenérgicos $\beta 2$ de acción prolongada, anticuerpos monoclonales anti-IgE (omalizumab y teofilina de liberación retardada) se utilizan de manera pautada, buscando estabilizar la enfermedad a largo plazo. Los segundos (agonistas adrenérgicos $\beta 2$ de acción corta inhalados y el bromuro de ipratropio), usados a demanda, actúan rápidamente para revertir la obstrucción bronquial y aliviar los síntomas. 


\section{Educación del paciente}

Como sucede con cualquier otra patología crónica, la colaboración del paciente constituye una pieza clave dentro de la estrategia terapéutica global. Se pretende que el enfermo conozca cuándo y cómo manejar los fármacos y las medidas preventivas, cuáles son las estrategias a seguir para evitar las exacerbaciones, qué hacer ante ellas y cuándo debe buscar ayuda médica. El plan permitirá al paciente tomar conciencia del estado de su proceso a través de la valoración de los síntomas, necesidades de medicación broncodilatadora y variaciones en el FEM. Los programas de educación específicos facilitan el aprendizaje de estas habilidades.

\section{Objetivos:}

1. Analizar el asma bronquial como un síndrome respiratorio que azota a la humanidad.

2. Describir la enfermedad, manifestaciones clínicas, fisiopatología, tratamiento y clasificaciones.

3. Analizar el comportamiento de esta enfermedad en el adulto mayor.

4. Relacionar el asma bronquial con el ejercicio físico.

\section{Desarrollo}

\section{Estadísticas en nuestro país}

En nuestro país, un estudio realizado en el municipio Guantánamo entre 2000 y 2001 en una empresa metalmecánica, encontró una prevalencia de $2 \%$ de asma ocupacional, aunque el $58,3 \%$ de los trabajadores presentó hiperreactividad bronquial, $25 \%$ tos, $14,2 \%$ disnea y $2,3 \%$ sibilancias 24 .

Otro estudio realizado en el Instituto Nacional de Salud de los Trabajadores (INSAT) sobre las enfermedades respiratorias diagnosticadas en el período 1988-2006, muestra resultados que se corresponden con las tendencias actuales reportadas a nivel internacional, donde los procesos alérgicos han superado a los procesos por exposición prolongada a polvos inorgánicos, las neumoconiosis, que pueden ser evitadas al disminuir la exposición en el medio ambiente laboral. Estas enfermedades fueron, en orden de frecuencia, las siguientes:

- Asma ocupacional: $12(42,8 \%)$

- Neumoconiosis: $9(32,1 \%)$

- Alveolitis alérgica extrínseca: 5 (17,8\%)

- Bronquitis ocupacional: $2(7,1 \%)$ 
El tiempo promedio de exposición para el asma ocupacional fue de 16 años, y de 12 para la bronquitis, existiendo necesariamente un tiempo de latencia de meses o años hasta que se produzca la sensibilización a la sustancia causal, tiempo que será menor en los individuos atópicos.

Otro dato recogido en el INSAT que puede llevarnos a dirigir nuestra mirada al problema del asma relacionada con el trabajo, son los casos de invalidez total (448 casos) por enfermedad respiratoria en nuestro país en el año 2007. En 8 provincias (Pinar del Río, Mayabeque, Artemisa, La Habana, Cienfuegos, Camagüey, Granma, Santiago y Guantánamo) más del 30 $\%$ de los casos eran asmáticos.

Actualmente se requiere un cambio en la organización de la actividad de seguridad y salud en el trabajo hacia un sistema de gestión de seguridad, salud y medio ambiente en el trabajo, que se ajuste a los cambios que se producen en la sociedad cubana. Es necesario crear una cultura relacionada con la seguridad del trabajador, con el cuidado de su salud y en la conservación del medio ambiente laboral, bajo el principio de que "la seguridad de todos es obligación de todos".

\section{Clasificaciones}

Según la clínica, la enfermedad se puede clasificar en cuatro grupos, dependiendo del estado basal del paciente, la frecuencia de las crisis, la función pulmonar y la variación.

\section{Asma intermitente:}

- Crisis de disnea breves, menos de una vez a la semana.

- Menos de dos dosis nocturnas al mes.

- Periodo intercrítico asintomático y con función normal (PEF o VEMS $>80 \%$ del teórico).

- Variabilidad $<20 \%$.

- Incluye el asma por ejercicio.

Asma persistente leve:

- Más de una crisis semanal, pero menos de una diaria.

- Más de dos crisis nocturnas al mes.

- A veces hay limitación de la actividad y del sueño. 
- Situación basal: PEF o VEMS >80\% del teórico.

- Variabilidad $>30 \%$.

Asma persistente moderada:

- Síntomas continuos.

- Más de una crisis nocturna a la semana.

- Limitación de la actividad y del sueño.

- Situación basal de PEF o VEMS 60-80\% del teórico.

- $\quad$ Variabilidad $>30 \%$.

Asma persistente grave:

- Síntomas continuos.

- Exacerbaciones y crisis nocturnas frecuentes y graves.

- Ingresos hospitalarios frecuentes.

- Limitación de la actividad y del sueño.

- Situación basal: PEF o VEMS <60\% del teórico.

- Variabilidad: $>30 \%$.

Esta clasificación es útil solo en pacientes no tratados. Sin embargo, resulta más adecuado establecer la gravedad teniendo en cuenta la medicación necesaria para controlar la enfermedad. Así, el asma grave es aquella que necesita un tratamiento de alta intensidad para conseguir el control.

Tabla 2. Características de los tipos de Asma

\begin{tabular}{|c|c|c|c|}
\hline Características & Controlada & $\begin{array}{c}\text { Parcialmente } \\
\text { controlada }\end{array}$ & No controlada \\
\hline Síntomas diurnos & $\begin{array}{c}\text { Dos o menos } \\
\text { veces por } \\
\text { semana }\end{array}$ & $\begin{array}{c}\text { Más de dos } \\
\text { veces por } \\
\text { semana }\end{array}$ & $\begin{array}{c}\text { Al menos tres veces por } \\
\text { semana }\end{array}$ \\
\hline
\end{tabular}


Vol. 2, N², p. 18-42, abril - junio, 2019

\begin{tabular}{|c|c|c|c|}
\hline $\begin{array}{c}\text { Uso de } \\
\text { medicación de } \\
\text { rescate }\end{array}$ & $\begin{array}{l}\text { Dos o menos } \\
\text { veces por } \\
\text { semana }\end{array}$ & $\begin{array}{l}\text { Más de dos } \\
\text { veces por } \\
\text { semana }\end{array}$ & $\begin{array}{c}\text { Parcialmente } \\
\text { controladas presentes en } \\
\text { una semana }\end{array}$ \\
\hline $\begin{array}{l}\text { Síntomas } \\
\text { nocturnos }\end{array}$ & Ninguno & Alguna vez & \\
\hline $\begin{array}{l}\text { Limitación de la } \\
\text { actividad }\end{array}$ & Ninguna & Alguna vez & \\
\hline $\begin{array}{c}\text { Función } \\
\text { pulmonar (FEV, } \\
\text { PEF) } \\
\end{array}$ & $\begin{array}{l}\text { Normal } \\
(>80 \%)\end{array}$ & $<80 \%$ & \\
\hline Exacerbaciones & Ninguna & $\begin{array}{c}\text { Una o más al } \\
\text { año }\end{array}$ & $\begin{array}{c}\text { Una exacerbación } \\
\text { convierte por definición } \\
\text { el asma en no } \\
\text { controlada. }\end{array}$ \\
\hline
\end{tabular}

Fuente: Elaboración propia.

\section{El asma por edades}

\section{Asma en edad pediátrica}

Cuando se trata de niños menores de 6 años, el análisis puede resultar difícil, esto se debe fundamentalmente, a que en estas edades las posibilidades diagnósticas son múltiples, el estudio de la función pulmonar resulta imposible de practicar de manera sistemática. Por consiguiente, en estos casos, el diagnóstico suele ser clínico, y se establece después de haber descartado otras afecciones que evolucionan con los mismos síntomas.

Aun así, existe un conjunto de niños cuya enfermedad es difícil de diferenciar, ya que durante los primeros años de vida presentan dificultades respiratorias y sibilancias en la evolución de las infecciones víricas respiratorias. Un grupo superará estos síntomas a partir de los 3; 4 o 5 años, mientras que el resto será el que, en realidad, se halle afecto por asma. Los antecedentes familiares y signos de atopía en el niño son datos que pueden orientar en uno u otro sentido.

En los niños, a cualquier edad, la tos seca e irritativa, de preferencia nocturna, en accesos, a menudo desencadenada por ejercicio y con la mala respuesta a los tratamientos habituales, puede ser el síntoma básico de la enfermedad.

\section{Asma en el embarazo}

Entre el 0.4 y el $1.3 \%$ de los embarazos ocurren en mujeres que padecen asma. En el $22 \%$ de las gestantes asmáticas el asma empeora-raramente en las últimas cuatro semanas de gestación o durante el parto-, mientras que un $29 \%$ mejora y en el $49 \%$ permanece invariable. 
Si el asma no es bien controlada durante el embarazo aumenta la probabilidad de una hipertensión gestacional, toxemia, parto prematuro, muerte perinatal y de crecimiento intrauterino retardado.

El factor desencadenante más frecuente en las gestantes son las infecciones respiratorias altas.

Los mecanismos de acción pueden incluir la hipoxemia materna que causa hipoxia fetal, interrupción del sueño debido a los frecuentes síntomas nocturnos, o una hiperventilación materna e hipocapnia que causa vasoconstricción de la placenta y reduce el flujo sanguíneo. Los ataques agudos de asma con frecuencia se acompañan de hipoxemia, deshidratación y desequilibrio ácido-básico, cada uno de los cuales puede afectar adversamente, al feto. Por tanto, el manejo del asma crónica debe incluir la detección y el tratamiento temprano de estos ataques. Un óptimo control del asma logra resultados del embarazo similares a los de la población no asmática.

\section{Asma y lactancia}

La mayor parte de los medicamentos antiasmáticos, tanto por vía inhalada como por vía oral o parental, se excretan por la leche materna en dosis muy bajas que, por lo general, no presentan problemas para los niños.

\section{Asma en el adulto}

La determinación de la gravedad del asma se consigue utilizando la información obtenida de la historia, así como del pico del flujo espiratorio. De esta manera, es posible determinar la gravedad clínica del asma.

El recuerdo del asma puede resultar poco fiable, debido a que depende del criterio y la memoria del paciente o de sus familiares; por ello se dice que es subjetivo. También depende, en cierto grado del criterio del equipo de salud. La evacuación de la gravedad del asma se debería realizar siempre antes de que el paciente inicie el tratamiento, y repetirse cuando el enfermo haya estado tomando su cantidad habitual de medicación durante un período prolongado.

\section{Asma ocupacional}

Se denomina asma ocupacional cuando se presenta relacionada, de manera causal, con la exposición en el ambiente de trabajo a polvos, gases, vapores o humos contenidos en el aire, o como enfermedad caracterizada por limitación variable al flujo de aire, con hiperreactividad bronquial o sin ella, por causas y trastornos atribuibles a un ambiente ocupacional particular y no a los estímulos fuera del sitio de trabajo. 


\section{Asma en el adulto mayor}

El asma en los ancianos suele presentarse sola o con otros problemas ventilatorios obstructivos, frecuentes a esta edad. Puede haber estado presente durante muchos años o ser recién diagnosticada. Puede ocurrir en fumadores activos o exfumadores.

En realidad, un no fumador con crisis de acortamiento de la respiración y jadeo, hace pensar más en asma que en la enfermedad pulmonar obstructiva crónica; pero el diagnóstico del asma no se debe descartar solo sobre la base del tabaquismo. De igual forma, la carencia de antecedentes de asma en la infancia no elimina esta posibilidad, aunque, ciertamente, parece que gran parte del asma en los ancianos no es una recurrencia de la conocida asma infantil.

Niveles de IgE séricos elevados y con una eosinofilia significativa en la sangre también hacen sospechar del asma y no de otras enfermedades obstructivas crónicas; pero una vez más, la falta de esos hallazgos no la descarta, incluso cuando la IgE absoluta no está en rango elevado. Debido a la variabilidad de los niveles de $\operatorname{IgE}$ en $\operatorname{los}$ ancianos, un valor clínico normal no es útil para excluir una enfermedad alérgica en una persona o cada individuo en particular.

La mayoría de los pacientes con asma, incluidos los ancianos, se presentan con jadeo. En los estudios de disnea se ha observado que este síntoma está más probablemente asociado al asma que a otras causas pulmonares o cardiacas de la disnea. Al reducir este síntoma, se debe estar consciente de que la percepción de una carga aumentada de resistencia parece reducida en los ancianos, por tanto, el paciente de edad avanzada puede reportar, erróneamente, la disnea, debido a la disminuida percepción de incrementos en la resistencia de las vías respiratorias. Otro motivo de informe erróneo en los ancianos es que a nivel de actividad es demasiado reducido para aumentar la demanda en el sistema ventilatorio; por consiguiente, hay menos ocasiones en que la disnea sea precipitada.

La espirometría es necesaria para definir la presencia y gravedad del trastorno obstructivo, así como las fluctuaciones en el grado de la obstrucción. Aunque a mayoría de los ancianos tienen cierto nivel de obstrucción fija, en especial sin presentar síntomas severos, hay una variabilidad bastante marcada en el volumen espiratorio forzado en el primer segundo. A menudo, la impresión clínica es que a medida que estos pacientes envejecen -finales de los 70 años-, existe menos fluctuación y un mayor elemento de obstrucción fija; las exacerbaciones ocurren, pero son menos frecuentes. El punto principal que se debe establecer es que el diagnóstico del asma no debe ser descartado sin una obstrucción fija en las vías respiratorias, con fluctuaciones bastante menores y después asociadas a un episodio bronquítico agudo precipitante. Incluso, en estos casos se está comenzando a negar a eliminar el asma de la lista de posibilidades diagnósticas. 
La experiencia clínica y los datos epidemiológicos existentes hacen cada vez más evidente que los individuos con asma tienen un índice mucho menor de disminución en la función pulmonar que los pacientes con enfermedad pulmonar obstructiva crónica.

Como los esteroides inhalados no presentan mucho riesgo para el paciente, frente a la posibilidad de mucho más beneficio si tiene asma, se está cada vez más inclinado a indicar al paciente una prueba de esteroides inhalados, a la vez que, periódicamente, se controla su función pulmonar.

En el pasado, no era partidario de aplicar una prueba de corticoesteroides orales, a menos que hubiera una evidencia considerable para el diagnóstico de asma (eosinofilia en la sangre, una respuesta significativa broncodilatadora, un volumen espiratorio forzado durante el primer segundo fluctuante y una radiografía torácica sin enfisema). El interés médico en los efectos secundarios del esteroide y la posible dificultad de descartar el medicamento, retardan el comienzo del tratamiento. Con los esteroides inhalados disponibles hoy día, aquellos temores han disminuido porque los efectos secundarios son prácticamente nulos. Por tanto, es posible una prueba con un buen control fisiológico. Sin embargo, pueden tardar varios meses antes de que los efectos beneficiosos de los esteroides inhalados se puedan comprobar. Tanto el paciente como el médico de la familia deben ser tolerantes durante este período.

\section{Tratamiento farmacológico}

Básicamente el enfoque en la atención y el cuidado es el mismo para cualquier asmático de cualquier edad, la función pulmonar debe ser controlada como parte del programa terapéutico. En el cuidado a largo plazo existen algunas precauciones que se deben tomar, simplemente, porque el paciente es un anciano y con frecuencia tiene más de un diagnóstico.

Algunos medicamentos, que son mucho más utilizados en el adulto mayor, pueden empeorar el asma. Hay que asegurarse de que el paciente no esté tomando betablonqueadores, por un problema cardiovascular o por un glaucoma; los inhibidores de la enzima conversora de la angiotensina pueden aumentar la tos, lo cual puede ser mal interpretado como una exacerbación del asma; los medicamentos antiinflamatorios no esteroides, que con frecuencia son prescritos para problemas de artritis en el anciano, pueden también ser desencadenantes de síntomas del asma. Además, debido a la enfermedad coexistente y los cambios con el envejecimiento, los ancianos tienen más posibilidades de mostrar efectos secundarios con el uso de los medicamentos antiasmáticos, comúnmente prescritos.

La terapia inicial del asma en los ancianos, al igual que en los individuos más jóvenes, es un agonista betainhalado. Lo fundamental es asegurar que el inhalador sea utilizado de forma adecuada. 
Con una instrucción apropiada y el uso del espaciador, la mayoría de los adultos mayores no requieren el empleo de un nebulizador de pequeño volumen para el aerosol. También se evitan los agonistas beta inespecíficos y con acción alfa y beta, debido a la creciente incidencia de los efectos secundarios, incluidos el nerviosismo, insomnio y agravamiento de las arritmias cardiacas. En Cuba se aconsejan a nuestros pacientes el uso de agonistas $\beta 2$ según sea necesario. Una gran parte de nuestros ancianos asmáticos han estado utilizando broncodilatadores inhalados por muchos años y, a menudo, se sienten confundidos de que el enfoque del tratamiento haya cambiado; los betaantagonistas son utilizados ahora "cuando es necesario", en lugar de serlo sobre una base regular.

Como muchos de estos pacientes también tienen bronquitis crónica, un número considerable demuestra una buena respuesta terapéutica al bromuro de ipatropio inhalado. Cuando un betaagonista y el ipatropio son prescritos, hay que asegurarse de que el paciente entiende qué inhalador debe ser utilizado regularmente y cuál cuando "sea necesario".

Si el paciente requiere una terapia regular con betaagonista (según la historia, los síntomas y las medidas objetivas de la función pulmonar) entonces, debe considerar la terapia con esteroides. Aunque en el pasado se instituyó una prueba con esteroides, y se utilizaba prednisona oral, en la actualidad, se utilizan esteroides inhalados, los cuales son muy efectivos, y pueden evitar los medicamentos orales.

En algunos casos, es necesario un tratamiento farmacológico adicional. Los medicamentos incluidos son teofilina, de acción sostenida y cubierta entérica, cromolina y antihistamínicos H1 de segunda generación. Para aquellos pacientes que muestren efectos secundarios (en su mayoría nerviosismo y síntomas gastrointestinales) se reducirá la toma o se descontinuará, en dependencia de la dosis inicial y la gravedad de dichos efectos, mientras que, a la vez, se controlan los cambios en los síntomas y se mide la función pulmonar objetiva.

Con frecuencia, la cromolina es prescrita para los asmáticos jóvenes, y aunque se pueden beneficiar algunos ancianos, rara vez es tan efectiva como los esteroides inhalados.

La función de la inmunoterapia en el tratamiento de los asmáticos longevos no está clara sin una buena evidencia de eficacia; existe la presunción de que las respuestas a la inmunoterapia son reducidas en estos pacientes y en aquellos no seniles con una función pulmonar basal deteriorada.

El objetivo del tratamiento en el asmático es lograr un nivel satisfactorio de la función, suficiente, pero sin dañar. Debido a que muchos pacientes tienen un nivel de obstrucción fija, la espirometría normal no es el objetivo. En algunas situaciones hemos observado que hay que aceptar un nivel ligeramente inferior del medicamento, aunque no máximo de la función para evitar efectos secundarios perjudiciales a causa de los agentes secundarios perjudiciales a causa de los agentes terapéuticos. 
La decisión de aceptar un rango dado de función, se debe tomar con el paciente después de discutir su forma de vida, objetivos y expectativas.

\section{Asma bronquial y ejercicio físico}

La presencia de síntomas desencadenados con el ejercicio en un paciente asmático puede indicar que el paciente no está bien controlado.

El diagnóstico se realizará demostrando una variación de al menos el 15\% del FEM o del FEV1 entre distintas medidas tomadas antes y después del ejercicio.

Para la prevención de la broncoconstricción inducida por el ejercicio son de elección betaagonistas de vida media corta. También podemos usar beta-agonistas de vida media larga, cromoglicato o nedocromil antes de la realización del ejercicio. Los antileukotrienos administrados de forma continua han demostrado protección efectiva frente al broncoespasmo inducido por el ejercicio.

Nuestro objetivo debe ser que el paciente pueda realizar cualquier tipo de ejercicio con total normalidad.

Para disminuir el uso de medicación, se aconseja realizar un calentamiento progresivo antes del ejercicio.

El asma inducida por el ejercicio se produce después del inicio de una actividad física vigorosa, se manifiesta como un cuadro de tos, disnea, dolor u opresión torácica, jadeos o agotamiento durante y después del ejercicio1 alcanzando su pico máximo de 5 a 10 minutos después de parar. Los síntomas a menudo se resuelven espontáneamente después de otros 20 o 30 minutos1.

La mayoría de los asmáticos pueden presentarla y en algunos casos será la única manifestación de la enfermedad. Es más frecuente en niños y adultos jóvenes. Sin embargo, su presencia puede indicar que el paciente no está bien controlado. En este caso, el paciente, podría responder simplemente con un tratamiento antiinflamatorio regular.

La confirmación se realiza midiendo de forma objetiva el problema. Hay que demostrar una reducción mayor del 15\% del FEM o del FEV1 tras la realización del ejercicio (test de carrera libre) (Ver capítulo de diagnóstico) En la inmensa mayoría de los pacientes, el broncoespasmo inducido por el ejercicio no deberá limitar ni la participación ni el éxito en ejercicios intensos. Con beta-2 agonistas se previene el asma inducida por el ejercicio en más del $80 \%$ de los pacientes1.

Las medidas terapéuticas recomendadas son: 
- De dos a cuatro puffs de un beta-2 agonista de corta duración 5 a 60 minutos antes del ejercicio, preferiblemente lo más cerca del comienzo del mismo. Los efectos de este tratamiento duran de 2 a 3 horas.

- Una inhalación de un beta-2 agonista de larga duración al menos 30 minutos antes del ejercicio. El efecto durará de 10 a 12 horas. Con el uso de Beta-2 de vida media larga se ha comprobado la existencia de tolerancia al cabo de varias semanas, con lo que se hace necesario aumentar la dosis para obtener los mismos resultados.

- Cromoglicato o nedocromil también pueden usarse antes del ejercicio, durando su acción de una a dos horas. El efecto del nedocromil parece más pronunciado en asma a esfuerzo más severo.

- Los antileukotrienos han demostrado proporcionar protección contra el broncoespasmo inducido por el ejercicio si se toman de forma continuada y no presentan el fenómeno de la tolerancia.

- Antes del ejercicio, un calentamiento de 6-10 minutos puede ayudar a los pacientes que puedan tolerar un ejercicio continuo con síntomas mínimos, así como a reducir la incidencia y severidad del asma. El precalentamiento puede prevenir el uso de repetir la medicación.

- Incrementar la medicación de base si los síntomas ocurren con actividades o ejercicios habituales.

- El control a largo plazo del asma con medicación antiinflamatoria como corticoides inhalados, cromoglicato o nedocromil, puede reducir la frecuencia y la severidad de broncoespasmo inducido por el ejercicio.

Hay que advertir a los profesores y a los entrenadores que el paciente padece de asma por el ejercicio1. Los pacientes con asma que participen en competiciones deberán comunicar la medicación que utilicen ya que pueden dar positivo en controles anti-doping.

Uno de los objetivos del control del asma es que la mayoría de los pacientes asmáticos sean capaces de participar en cualquier actividad física que elijan sin experimentar síntomas. Además, la actividad física será parte del tratamiento de los pacientes con asma inducida por ejercicio.

El Asma por ejercicio consiste en la aparición de espasmos bronquiales cuando se realizan actividades físico-deportivas. Es una forma de asma muy frecuente entre los que padecen la enfermedad y también en pacientes sin asma, pero con alguna enfermedad alérgica e incluso en ocasiones se observa en personas sin afecciones alérgicas. Lo importante es que en estos 
casos igual se puede practicar deportes, ya que hoy hay medicamentos que, tomados previamente al deporte, impiden que aparezca el broncoespasmo.

Un mito que lamentablemente aún existe y que debemos combatir es: "Los niños y adultos que padecen asma no pueden practicar deportes". Hoy sabemos que el paciente con asma puede y debe practicar deportes, como parte de su tratamiento. Con un control adecuado prácticamente todos los asmáticos pueden desarrollar cualquier tipo de actividad física.

Los pacientes asmáticos pueden realizar ejercicios físicos, e incluso pueden destacarse en varias disciplinas sin tener que ser un atleta profesional, el hecho de estar activo y hacer deporte puede beneficiar mucho la salud de estos pacientes, se mantiene el cuerpo en forma, mantener el peso corporal adecuado, incluso fortalecer los músculos que participan en la respiración, esto contribuye a que los pulmones funcionen mejor.

El ejercicio físico tiene fantásticos efectos beneficiosos sobre la salud emocional, al hacer ejercicios el cuerpo produce endorfinas, sustancias químicas que hacen que la persona se sienta más tranquila y feliz. Los deportes ayudan a algunas personas a dormir mejor y puede ayudar con los problemas psicológicos como la depresión leve.

Hay algunos deportes que no tienen tantas posibilidades de ocasionar problemas a la gente que padece asma. Por ejemplo, la natación, el esquí de pista tienen menos posibilidades de desencadenar crisis asmáticas. Deportes como el béisbol, el futbol americano, la gimnasia y el atletismo (carreras de velocidad, salto de obstáculos, lanzamiento de jabalina, etc., excluyendo las actividades de resistencia y/o largo recorrido) también suelen ser adecuados para las personas con asma.

Los deportes de resistencia, como correr trayectos largos y el ciclismo, y aquellos que requieren hacer un gasto energético durante un periodo de tiempo prolongado, como el futbol y el baloncesto pueden ser menos recomendables para una persona con asma. Esto es especialmente cierto para los deportes que se practican en condiciones de frio como el esquí de travesía o el hockey sobre hielo. Pero esto no significa que se tenga que renunciar a esos deportes.

\section{Factores que estimulan el asma}

- Sustancias que producen alergia: polen, polvo, alimentos y medicamentos.

- Resfriado y gripe.

- Estrés emocional.

- Ejercicio físico inadecuado.

- Perfumes, humo de tabaco, gases, etc.

- Lluvia, frio, calor y cambios bruscos de temperatura. 
En una persona con asma bronquial, un ejercicio físico o deporte adecuado contribuye de una forma positiva, no sólo desde el punto de vista médico, sino psicológico. Es indispensable saber el grado de asma y su respuesta con el ejercicio, para poder actuar con medidas profilácticas si fuera necesario, y para que los adultos (incluso los profesionales) no limiten indebidamente las actividades físicas. El ejercicio físico en el paciente puede realizarse aun cuando el paciente se encuentre recibiendo tratamiento medicamentoso, ya que ambos son beneficiosos. En algunas ocasiones los ejercicios respiratorios, al comienzo, pueden producir mareo en el paciente. Este fenómeno no tiene importancia alguna y basta con esperar unos breves momentos para que el mismo desaparezca. Las actividades deportivas, programadas y dosificadas adecuadamente, ejercen un efecto positivo en el tratamiento del asma tanto en el punto de vista psíquico como físico.

\section{Precauciones a adoptar}

- El paciente debe tener bien controlada su asma antes de comenzar a realizar ejercicios físicos.

- Llevar siempre encima la medicación de rescate.

- Consultar con su médico de familia acerca de los planes para entrenar y practicar deporte.

- Evitar entrenamientos al aire libre cuando los niveles de polen y esporas sean altos, llevar bufanda o un pasamontaña cuando se entrene en el invierno.

- Tomar el aire por la nariz y expulsarlo por la boca durante el entrenamiento.

- Dedicarle tiempo al calentamiento antes de comenzar el entrenamiento y al enfriamiento al acabar la sesión.

- Asegurarse de que el entrenador y los compañeros de equipo conozcan acerca de que padece asma y los pasos a seguir en caso de presentarse una crisis.

- Qué hacer en caso de crisis durante el ejercicio físico

- Parar el ejercicio.

- Procurar actuar con calma. Si se han realizado las normas básicas la crisis no será muy intensa.

- Permitir que se pueda respirar bien. No situarse alrededor del compañero que tiene la crisis.

- Administrar la medicación broncodilatadora lo antes posible.

- Iniciar la respiración silbante (fruncir los labios como si se fuera a silbar). Permite que el aire salga de los pulmones y evita la hiperventilación y la insuflación del tórax.

- Cuando se solucione la crisis puede incorporarse al ejercicio, si no se está muy cansado, pero bajando la intensidad de la actividad.

- Al finalizar el ejercicio realizar una medición de FEM, para ver cómo se encuentra. Si está en la zona amarilla tomar otra dosis de broncodilatador. 
- Si no se recupera y está en zona roja tomar otra dosis de broncodilatador y esperar 5 minutos. Medir nuevamente el FEM. Si no se aumenta consultar un médico o ir a un centro asistencial.

\section{Deportistas asmáticos}

El caso más famoso es el del nadador estadounidense Mark Spitz, quien a pesar de sufrir esta enfermedad logró colgarse siete medallas doradas durante los Juegos Olímpicos de Münich en 1972.

Otro caso importante es el del futbolista del Manchester United Paul Scholes fue referente del fútbol británico durante una década y fue capaz de superar esta afección, que le había sido diagnosticada a los 21 años.

En los Juegos Olímpicos de Barcelona 1992, entre el 8 y el 12\% de los competidores eran asmáticos.

El argentino Abelardo Sztrum, en remo, Gabriel Simón, record argentino en carrera de 100 m, Eric Pedercini, medalla de oro en Gimnasia en aparatos en Winnipeg en 1999.

La corredora británica Paula Radcliffe, quien ha ganado varias competencias alrededor del mundo, incluyendo la maratón de Londres. Según Redcliffe, "lo más importante es el calentamiento; es fundamental hacer algo de estiramiento y correr carreras lentas para acondicionar el cuerpo". Ella realiza una sesión previa a la carrera de aproximadamente 45 minutos y también hace 15 minutos de trote antes de cada competencia. "Lo ideal es controlar la respiración durante el calentamiento para crear un ambiente ideal a la hora de practicar la actividad física".

Sin embargo, correr o jugar futbol no son los mejores deportes para las personas con asma. Una de las prácticas más recomendadas para mejorar el rendimiento físico, inclusive por personas como Radcliffe o Scholes, es la natación.

Para la farmacéutica española Antonia Mangues y la enfermera María Pilar García, esta es la mejor opción para quienes sufren de esta dolencia respiratoria. "Generalmente nada es mejor que correr. Efectivamente, la natación es uno de los mejores deportes para los asmáticos, porque provoca excesiva presión pectoral y se practica en ambiente húmedo". Es aconsejable que se tenga en cuenta que seis minutos de deporte pueden producir un ataque de asma, por lo que recomiendan practicar en intervalos de menor duración. "Las carreras de relevos o los deportes de equipo son muy indicados para los asmáticos. Un tratamiento con base correcta mejora el asma y la tolerancia al ejercicio".

No solo la natación o las carreras por relevos son buenos para las personas con asma, de acurdo al médico deportivo John Bottrell, las artes marciales son un ejercicio adecuado 
debido a que se practica bajo techo y, por lo general, con movimientos cortos que permiten a las personas estar en forma y aumentar masa muscular. También el trabajo con pesas sirve para varias cosas: aumenta la masa muscular y perder grasa corporal. Otros ejercicios recomendables son el ciclismo y caminar.

El ejercicio enseña y ayuda a vivir mejor con el asma. A través del ejercicio el paciente con asma realizará actividades físicas con las que obtendrá por sí mismo la confianza y entusiasmo necesario para tomar parte en actividades a las que está expuesto diariamente.

\section{Los beneficios de la práctica de la actividad física y el juego en el niño y el adulto son:}

1. Facilitar y permitir el correcto desarrollo físico y psíquico.

2. Favorecer la integración en el grupo.

3. Mejorar la autoestima del individuo adolescente.

4. Mejorar la condición física en general.

5. Permitir una mayor tolerancia al ejercicio.

6. Las crisis en caso de aparecer, lo hacen para trabajos mucho más intensos.

7. Permite un mayor control de las crisis.

8. Todo ello nos lleva a un mejor conocimiento del asma y a un establecimiento de unas dosis de medicamento más ajustadas.

Actitud práctica Siempre, antes de iniciarse en la actividad física debe consultarse con el especialista cual es la medicación idónea personal para evitar la crisis. Con independencia del tratamiento de la enfermedad, en el asma de esfuerzo se administra lo siguiente:

1. Lo habitual es, previo al ejercicio, inhalar un broncodilatador. También se puede administrar Nedocromil o Cromoglicatoque es igual de efectivo en la mayoría de los asmáticos.

2. En el caso que el broncodilatador o el nedocromil no acaben de evitar las crisis (y se ha descartado que se halle en un periodo inestable de su asma) se pueden administrar juntos antes del ejercicio. Con ello se evita la crisis en casi el 100\% de los casos.

Para hacer un ejercicio el asmático debe valorar la condición física previa y estar en un periodo controlado de su asma. Una medida para valorarlo es mediante el control del flujo espiratorio máximo (FEM). Debe tomarse la medición siempre antes de iniciar una actividad física, para controlar el estado de las vías aéreas.

\section{Preguntas habituales relacionadas con el asma}

\section{¿Se cura el asma?}


El asma es una enfermedad crónica que no tiene curación, pero el control de los factores desencadenantes, la instauración de un tratamiento precoz y el control del asma son los responsables de la desaparición de los síntomas y del buen pronóstico a largo plazo.

\section{¿Es verdad que los niños que toman corticoides inhalados se quedan pequeños?}

Diversos estudios indican que los niños que toman corticoides inhalados a dosis altas pueden ver disminuida su velocidad de crecimiento en aproximadamente $1 \mathrm{~cm}$ al año, pero esto no influirá en su talla final. Pero es excepcional que un niño tenga que tomar dosis muy altas de corticoides. Lo habitual es que se controlen muy bien con dosis bajas o asociando otros fármacos a las dosis bajas de corticoides inhalados.

\section{¿Cuánto tiempo dura el tratamiento?}

El asma es una enfermedad crónica y por lo tanto no nos podemos poner plazos en su tratamiento. El objetivo que nos marcamos es conseguir un buen control del mismo, evitando los síntomas y las crisis, en el menor tiempo posible, con los menores efectos secundarios y con la menor dosis de medicación

\section{¿El tratamiento me seguirá haciendo el mismo efecto si utilizo los inhaladores diariamente?}

Sí. La utilización diaria de los inhaladores no le perjudica, tampoco hace que la medicación pierda efecto, sino que además le permite que, para controlar su asma, la cantidad de medicación necesaria sea cada vez menor.

\section{Tengo asma ¿algún día podría acabar padeciendo un asma de difícil control?}

Aunque el asma es una enfermedad imprevisible, es muy difícil que una persona con asma desde hace tiempo y que sigue un tratamiento adecuado, lo pueda desarrollar. Aunque no existe un acuerdo unánime al respecto, parece ser que las auténticas formas graves de asma acostumbran a presentarse ya desde el inicio de la enfermedad, no a lo largo de su curso evolutivo.

\section{Creo que tengo un asma de difícil control ¿qué hago?}

Primero, no desesperar. Difícil control, no significa imposible, sólo que cuesta más. Segundo, pacientes que al principio parecía que tenían un asma difícil, luego tras un tratamiento y educación adecuados, no lo eran realmente. Tercero, posiblemente su caso debería ser analizado en una unidad especializada en asma.

¿El asma, es una enfermedad psicológica? 
No, el asma no es una enfermedad psicológica, pero la ansiedad o la depresión pueden incrementar una mayor presencia de síntomas y una peor evolución de la enfermedad, precisando más medicación o algunas veces el soporte de un psicólogo. También puede alterar la percepción de los síntomas del asma, por ejemplo, el ahogo, con lo cual el paciente no reconoce de la aparición de una crisis.

\section{Conclusiones.}

- El asma es un síndrome respiratorio caracterizado por una disminución de la luz bronquial.

- El asma afecta a todas las edades, puede aparecer en cualquier etapa de la vida y es más común en los niños.

- El ejercicio físico moderado mejora las crisis de asma, si este es muy prolongado puede conllevar a una crisis.

\section{Referencias bibliográficas.}

Hinshaw C, LH Garland. Enfermedades del tórax. PP. 228-45, Edic. Revolucionaria. La Habana, 1968.

Canete Ramos C; A Llunell Casanovas; B Rodríguez Sanchón; F Marnesa Presas. Asma bronquial, en neumología. PP. 35-55, Ed CEA, Barcelona, 1977.

Guía Española para el manejo del Asma (GEMA) 2009. Arch Broconeumol. 2009; 45 Supl $7: 1-35$.

GINA 2006. Global Strategy for Asthma Management and Prevention NHLBI/WHO Workshop Report, 2006. Disponible en www.ginasthma.com

BTS 2007. British Thoracic Society, Scotish Intercollegiate Guidelines Network. British guideline on the management of asthma. 2007. Thorax. 2008; 63 Suppl 4:iv1-121. Disponible en www.sign.ac.uk/guidelines.

Anderson GP. Endotyping asthma: new insights into key pathogenic mechanism in a complex, heterogeneous disease. Lancet. 2008; 372:107-19.

Beasley R, Crane J, Lai CKW, Pearce N. Prevelance and etiology of asthma. J Allergy Clin Immunol. 2000;105:S466-72.

Viegi G, Annesi I, Mattelli G. Epidemiology of asthma. Eur Respir Mon. 2003;23:1-25.

Lai CKV, Beasley R, Crane J, Foluaki S, Shah J, Weiland S. Global variation in the prevalence and severity of asthma symptoms: Phase Three of the International Study of asthma and Allergies in Childhood (ISAAC). Thorax 2009;64:476-83. 
Perpiñá Tordera M, de Diego Damiá A. Asma. En: Villasante C, editor. Enfermedades respiratorias. Madrid: Aula Médica; 2002. P. 459-69.

Perpiñá Tordera M, Lloris Bayo A. Hiperrespuesta bronquial. Concepto y mecanismos patogénicos. En: Pino García JM, García Río F, editores, Estudio de la función pulmonar. Hiperrespuesta bronquial. Madrid: Sanitaria 2000; 2006. P 7-36.

Castillo Laita JA, Torregrosa Bertet MJ y Grupo de Vías Respiratorias. Guía Rápida del Protocolo de Diagnóstico de Asma. Disponible en www.aepap.org/gvr/protocolos.htm

Rodríguez-Rodríguez M. Registros de flujo espiratorio máximo. En: Delgado J, Quirce S, editores. Pruebas de función pulmonar e inflamación bronquial en el asma. Madrid: GSK; 2012. P 37-53.

Recomendaciones y algoritmos de práctica clínica de la Sociedad Española de Alergología e Inmunología Clínica Asma. Comité de Asma de la SEAIC 2010. Madrid. Luzán 5, S.1.; 2010 .

Métodos de diagnóstico en las enfermedades alérgicas. En: Peláez A, Dávila IJ, editores. Tratado de Alergología de la Sociedad Española de Alergología e Inmunología Clínica SEAIC. Madrid: Ergón; 2007. 115-223.

Global Iniciative for Asthma. Global strategy for asthma management and prevention NHLBI/WHO Workshop Report 2006. Disponible en www.ginasthma.com

Área de asma de la Sociedad Española de Neumología y Cirugía Torácica (SEPAR) GEMA 2009. Guía española para el manejo del asma. Madrid: Luzan 5, S.A; 2009. Disponible en www.gemasma.com

López Viña A, Agüero Balbín R, Aller Álvarez JL, Bazús Gonzáles T, García cosio FB, de Diego Damiá A,et al. Normativa para el asma de control difícil. Arch Bronconeumol. 2005; 41:513-23.

Bousquet J, Cuwenberge P, Khaltaer N, ARIA Workshop Group NOrld health Organizationl. Allergic rhinitis and its impact on astma J Allergy Clin Immunol. 2009; 108:S147334.

Leggett JJ, Johnston BT, Mills M, Gamble J, Heaney LG. Prevalence of gastroesophageal reflux in difficult asthma: relationship to asthma outcome. Chest. 2005;127:1907-98.

Ten Brinke A, Sterk PJ, Masclee AA, Spinhoven P, Schmidt JT, Zwinderman AH, et al. Risk factors of frequent exacerbations in difficult to treat asthma. Eur Respir $\mathrm{J}$. 2005;26;812-8. 
PARA CITAR EL ARTÍCULO INDEXADO.

Mederos González, A., \& Herrera Fraga, E. (2019). Asma bronquial y ejercicio físico. Manifestaciones en el adulto mayor. Anatomía Digital, 2(2), 18-42. https://doi.org/10.33262/anatomiadigital.v2i2.1079

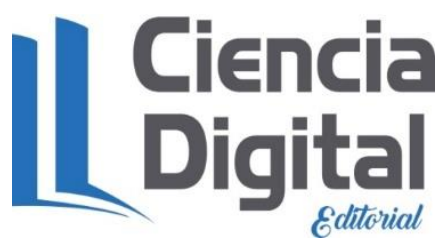

El artículo que se publica es de exclusiva responsabilidad de los autores y no necesariamente reflejan el pensamiento de la Revista Anatomía Digital.

El artículo queda en propiedad de la revista y, por tanto, su publicación parcial y/o total en otro medio tiene que ser autorizado por el director de la Revista Anatomía Digital.
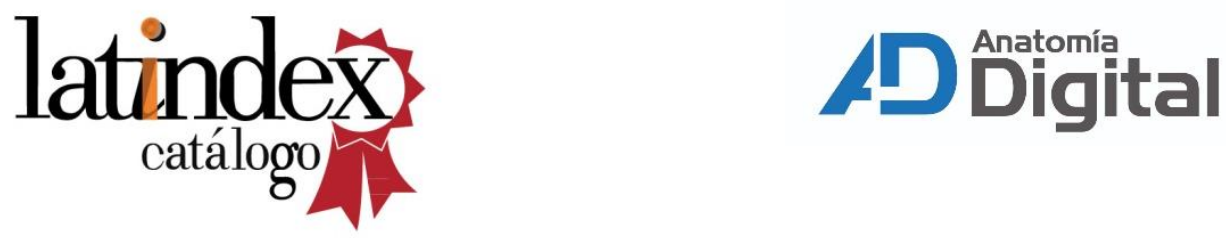